\title{
Fuzzy Fixed Point Theorems for Fuzzy Mappings Viafuzzy $\beta$-Admissible
}

\author{
Buthainah A. A. Ahmed ${ }^{1}$, Manar Falih Dheyab ${ }^{2}$ \\ Department of Mathematics, College of Science, University of Baghdad, Iraq
}

\begin{abstract}
The aim of this paper is introduced the notion offuzzy $\beta$-admissible, fuzzy $\beta$-Jaggi contractive and study some results of fuzzy fixed point for fuzzy mapping via fuzzy $\beta$-admissible in Hilbert spaces.
\end{abstract}

Keywords: fuzzy mapping, fuzzy fixed point , $\beta$ - admissiblemapping and Hilbert space

\section{Introduction}

The concept of fuzzy set was introduce by L.Zadeh [3]in 1965.After that a lot of work has been done regarding fuzzy set and fuzzy mappings. The concept of fuzzy mapping was first introduced by Heilpern [4].In 2001 , Estruch and Vidal [5] proved a fuzzy fixed point theorem for fuzzy contractive mappings. On the other hand, the concept of an $\beta-$ admissible mapping was introduced by Samet et al.[2]. Recently, Mohammadi et al. [1] introduced the concept of $\beta$-admissible for multivalued mappings . In this paper, introduced fuzzy $\beta$ - admissible mapping, fuzzy $\beta-$ Jaggi contractive mappingand study some results of fuzzy fixed point theorems for fuzzy mapping via fuzzy $\beta-$ admissible in Hilbert space.

\section{Preliminaries}

In this section, we recall some basic definitions and preliminaries that will be needed in this paper.

Definition 2.1[3]: Let $\mathrm{H}$ be a Hilbert space and $\mathrm{F}(\mathrm{H})$ be a collection of all fuzzy sets in $H$. Let $A \in F(H)$ and $\alpha \in[0,1]$ the $\alpha$ - level set of $A$, denoted by $A_{\alpha}$ is defined by

$$
\begin{aligned}
& \mathrm{A}_{\alpha}=\{\mathrm{x}: \mathrm{A}(\mathrm{x}) \geq \alpha\} \text { if } \alpha \in[0,1] \\
& \mathrm{A}_{0}=\{\mathrm{x}: \mathrm{A}(\mathrm{x})>\alpha\}
\end{aligned}
$$

Where $\overline{\mathrm{B}}$ denotes the closure of a set $\mathrm{B}$.

Definition 2.2[4]:A fuzzy set A is said to be an approximate quantity if and only if $A_{\alpha}$ is compact and convex for each $\alpha \in[0,1]$, and $\sup _{\mathrm{x} \in \mathrm{X}} \mathrm{A}(\mathrm{x})=1$. When $\mathrm{A}$ is an approximate quantity and $\mathrm{A}\left(\mathrm{x}_{0}\right)=1$ for $\operatorname{somex}_{0} \in \mathrm{H}, \mathrm{A}$ is identified with an approximate of $\mathrm{x}_{0}$.

The collection of all fuzzy sets in $\mathrm{H}$ is denoted by $\mathrm{F}(\mathrm{H})$ and $\mathrm{W}(\mathrm{H})$ is the sub collection of all approximate quantities.

Definition 2.3[4]: Let $A, B \in W(H)$ and $\alpha \in[0,1]$. Then

1) $P_{\alpha}(A, B)=\inf _{x \in A_{\alpha}, y \in B_{\alpha}}\|x-y\|$

2) $D_{\alpha}(A, B)=\operatorname{dis}\left(A_{\alpha}, B_{\alpha}\right)$, where "dis" is the Hausdorff distance

3) $\mathrm{D}(\mathrm{A}, \mathrm{B})=\sup _{\alpha} \mathrm{D}_{\alpha}(\mathrm{A}, \mathrm{B})$

4) $\mathrm{P}(\mathrm{A}, \mathrm{B})=\sup _{\alpha} \mathrm{P}_{\alpha}(\mathrm{A}, \mathrm{B})$.

It is to be noted that for any ' $\alpha$ ', $P_{\alpha}$ is a non decreasing as well as continuous function.
Definition 2.4[3]. Let A, B $\in \mathrm{W}(\mathrm{H})$. An approximate quantity $\mathrm{A}$ is said to be more accurate than $\mathrm{B}$ (denoted by $\mathrm{A} \subset \mathrm{B})$ if and only if $\mathrm{A}(\mathrm{x}) \leq \mathrm{B}(\mathrm{x}), \forall \mathrm{x} \in \mathrm{H}$.

Definition 2.5[4]:A mapping $\mathrm{T}$ from the set $\mathrm{H}$ into $\mathrm{W}(\mathrm{H})$ is said to be fuzzy mapping.

Definition 2.6[4]: The point $x \in H$ is called fixed point for the fuzzy mapping $\mathrm{T}$ if $\{\mathrm{x}\} \subset \mathrm{Tx}$. If $\mathrm{x}_{\alpha} \subset \mathrm{Tx}$ is called fuzzy fixed point of $\mathrm{T}$. We shall use the following lemmas due to Helipern.

Lemma 2.7[4]: $\mathrm{P}_{\alpha}(\mathrm{x}, \mathrm{B}) \leq\|\mathrm{x}-\mathrm{y}\|+\mathrm{P}_{\alpha}(\mathrm{y}, \mathrm{B}), \forall \mathrm{x}, \mathrm{y} \in \mathrm{H}$.

Lemma 2.8[4]:If $\left\{\mathrm{x}_{0}\right\} \subset \mathrm{A}$, then $\mathrm{P}_{\alpha}\left(\mathrm{x}_{0}, \mathrm{~B}\right)$ $\leq \mathrm{D}_{\alpha}(\mathrm{A}, \mathrm{B}), \forall \mathrm{B} \in \mathrm{W}(\mathrm{H})$.

Lemma 2.9[4]:Let $A \in W(H)$ and $x_{0} \in H$, if $\left\{x_{0}\right\} \subset$ A then $\mathrm{P} \alpha(\mathrm{x} 0, \mathrm{~A})=0$, for each $\alpha \in 0,1$.

Lemma 2.10[4]: Let $H$ be a Hilbert space and $T$ fuzzy mapping from $H$ into $W(H)$ and $x_{0} \in H$, then there exist $\mathrm{x}_{1} \in \mathrm{H}$ such that $\left\{\mathrm{x}_{1}\right\} \subset \mathrm{Tx}_{0}$.

\section{Fuzzy Fixed Point Theorem}

In this section, we introduce the concept of fuzzy $\beta-$ admissiblefor fuzzy mapping and some results of fuzzy fixed point theorems.

Definition 3.1[2]: Let $\mathrm{H}$ be a Hilbert space , $\beta: \mathrm{H} \times \mathrm{H} \rightarrow$ $[0, \infty), \alpha \in[0,1]$ and $\mathrm{T}: \mathrm{H} \rightarrow \mathrm{W}(\mathrm{H})$. A mapping $\mathrm{T}$ is said to be fuzzy $\beta-$ admissible if for each $\mathrm{x} \in \mathrm{H}$ and $\mathrm{y} \in[\mathrm{Tx}]_{\alpha}$, with $\beta(x, y) \geq 1$, we have $\beta(y, z) \geq 1$ for all $\mathrm{z} \in[\mathrm{Ty}]_{\alpha}$.

Definition 3.2:Let $\mathrm{H}$ be a Hilbert space , $\beta: \mathrm{H} \times \mathrm{H} \rightarrow$ $[0, \infty), \alpha \in[0,1]$ and $\mathrm{T}: \mathrm{H} \rightarrow \mathrm{W}(\mathrm{H})$. A mapping $\mathrm{T}$ is said to be fuzzy $\beta^{*}-$ admissible if for each $\mathrm{x}, \mathrm{y} \in \mathrm{H}$ with $\beta(\mathrm{x}, \mathrm{y}) \geq$ 1 , we have $\beta\left([\mathrm{Tx}]_{\alpha},[\mathrm{Ty}]_{\alpha}\right) \geq 1$

Where $\beta\left([\mathrm{Tx}]_{\alpha},[\mathrm{Ty}]_{\alpha}\right)=\inf \left\{\beta(\mathrm{a}, \mathrm{b}): \mathrm{a} \in[\mathrm{Tx}]_{\alpha}\right.$ and $\mathrm{b} \in$ Ty $\alpha$.

Remark 3.3: If $\mathrm{T}$ is fuzzy $\beta^{*}-$ admissible, then Tis also fuzzy $\beta-$ admissible mapping . 


\section{International Journal of Science and Research (IJSR) \\ ISSN (Online): 2319-7064}

Index Copernicus Value (2015): 78.96 | Impact Factor (2015): 6.391

Definition 3.4: Let $\mathrm{H}$ be a Hilbert space, $\beta: \mathrm{H} \times \mathrm{H} \rightarrow[0, \infty)$. A fuzzy mapping $\mathrm{T}: \mathrm{H} \rightarrow \mathrm{W}(\mathrm{H})$ is called fuzzy $\beta-$ Jaggi contractive, if there exists two function $\beta: \mathrm{H} \times \mathrm{H} \rightarrow$ $[0, \infty)$ and $\Psi:[0, \infty) \rightarrow[0, \infty)$, where $\Psi$ is non-decreasing and $\quad \sum_{\mathrm{n}=1}^{\infty} \Psi^{\mathrm{n}}(\mathrm{t})<\infty$, for each $t>0$ and $\Psi^{\mathrm{n}}$ is $\mathrm{n}-$ th iteration of $\Psi$ such that $\beta(\mathrm{x}, \mathrm{y}) \mathrm{D}^{2}(\mathrm{Tx}, \mathrm{Ty}) \leq$ $\Psi(\mathrm{M}(\mathrm{x}, \mathrm{y}))+\mathrm{LN}(\mathrm{x}, \mathrm{y}), \forall \mathrm{x}, \mathrm{y} \in \mathrm{H}$, where $\mathrm{L} \geq 0, \alpha \in$ $[0,1], \beta>0$,

$$
\begin{aligned}
& \mathrm{M}(\mathrm{x}, \mathrm{y})=\frac{\alpha \mathrm{P}_{\alpha}^{2}(\mathrm{x}, \mathrm{Tx}) . \mathrm{P}_{\alpha}^{2}(\mathrm{y}, \mathrm{Ty})}{\|\mathrm{x}-\mathrm{y}\|^{2}}+\beta\|\mathrm{x}-\mathrm{y}\|^{2}-\alpha \mathrm{P}_{\alpha}^{2}(\mathrm{x}, \mathrm{Tx}) \\
& \text { And } \mathrm{N}(\mathrm{x}, \mathrm{y}) \\
& \min \left\{\mathrm{P}_{\alpha}^{2}(\mathrm{x}, \mathrm{T} \mathrm{x}), \mathrm{P}_{\alpha}^{2}(\mathrm{y}, \mathrm{Ty}), \mathrm{P}_{\alpha}^{2}(\mathrm{x}, \mathrm{Ty}), \mathrm{P}_{\alpha}^{2}(\mathrm{y}, \mathrm{T} \mathrm{x})\right\} .
\end{aligned}
$$

Theorem 3.4: Let $\mathrm{H}$ be a Hilbert space and $\mathrm{T}$ be a fuzzy $\beta$ Jaggi contractive mapping satisfies the following conditions:

1) $\mathrm{T}$ is fuzzy $\beta-$ admissible

2) There exists $x_{0} \in H$ and $x_{1} \in\left[T x_{0}\right]_{\alpha}$ such that $\beta\left(x_{0}, x_{1}\right) \geq 1$

3) If $\left\{x_{n}\right\}$ is a sequence in $H$ such that $\beta\left(x_{n}, x_{n+1}\right) \geq 1$ and $x_{n} \rightarrow u$ as $n \rightarrow \infty$, then $\beta\left(x_{n}, u\right) \geq 1$

4) $\Psi$ is continuous

Then, there exists $\mathrm{x} \in \mathrm{H}$ such that $\mathrm{x}_{\alpha}$ is a fuzzy fixed point of $\mathrm{T}$.

Proof: Let $\mathrm{x}_{0} \in \mathrm{H}$ and $\mathrm{x}_{1} \in\left[\mathrm{T}_{0}\right]_{\alpha}$ by condition (2) $\left(\mathrm{x}_{0}, \mathrm{x}_{1}\right) \geq 1$.

Since $\left[\mathrm{T} \mathrm{x}_{1}\right]_{\alpha}$ is non-empty compact subset of $\mathrm{H}$, there exists $\mathrm{x}_{2} \in\left[\mathrm{T} \mathrm{x}_{1}\right]_{\alpha}$, such that $\left\|\mathrm{x}_{1}-\mathrm{x}_{2}\right\|^{2}=\mathrm{P}_{\alpha}^{2}\left(\mathrm{x}_{1}, \mathrm{~T} \mathrm{x}_{1}\right) \leq$ $\mathrm{D}^{2}\left(\mathrm{~T} \mathrm{x}_{0}, \mathrm{~T} \mathrm{x}_{1}\right)$

$$
\text { So }\left\|\mathrm{x}_{1}-\mathrm{x}_{2}\right\|^{2} \leq \mathrm{D}^{2}\left(\mathrm{~T} \mathrm{x}_{0}, \mathrm{~T}_{1}\right)
$$

Since $\left(\mathrm{x}_{0}, \mathrm{x}_{1}\right) \geq 1$, then $\left\|\mathrm{x}_{1}-\mathrm{x}_{2}\right\|^{2} \leq \mathrm{D}^{2}\left(\mathrm{~T} \mathrm{x_{0 }}, \mathrm{T} \mathrm{x}_{1}\right)$

$$
\leq \beta\left(x_{0}, x_{1}\right) D^{2}\left(T x_{0}, T x_{1}\right) \leq \Psi\left(M\left(x_{0}, x_{1}\right)\right)+
$$

$\operatorname{Lmin}\left\{\mathrm{P}_{\alpha}^{2}\left(\mathrm{x}_{0}, \mathrm{~T} \mathrm{x}_{0}\right), \mathrm{P}_{\alpha}^{2}\left(\mathrm{x}_{1}, \mathrm{~T} \mathrm{x}_{1}\right), \mathrm{P}_{\alpha}^{2}\left(\mathrm{x}_{0}, \mathrm{~T} \mathrm{x_{1 }}\right), \mathrm{P}_{\alpha}^{2}\left(\mathrm{x}_{1}, \mathrm{~T} \mathrm{x}_{0}\right)\right\}$

$\leq \Psi\left(\mathrm{M}\left(\mathrm{x}_{0}, \mathrm{x}_{1}\right)\right)$

$+\operatorname{Lmin}\left\{\mathrm{P}_{\alpha}^{2}\left(\mathrm{x}_{0}, \mathrm{x}_{1}\right), \mathrm{P}_{\alpha}^{2}\left(\mathrm{x}_{1}, \mathrm{x}_{2}\right), \mathrm{P}_{\alpha}^{2}\left(\mathrm{x}_{0}, \mathrm{x}_{2}\right), \mathrm{P}_{\alpha}^{2}\left(\mathrm{x}_{1}, \mathrm{x}_{1}\right)\right\}$ $\left\|\mathrm{x}_{1}-\mathrm{x}_{2}\right\|^{2} \leq \Psi\left(\mathrm{M}\left(\mathrm{x}_{0}, \mathrm{x}_{1}\right)\right)$.

By the same argument, for $\mathrm{x}_{2} \in \mathrm{H}$ we have $\left[\mathrm{T} \mathrm{x}_{2}\right]_{\alpha}$ which is non-empty compact subset of $H$ and there exists $x_{3} \in$ $\left[\mathrm{T} \mathrm{x}_{2}\right]_{\alpha}$, such that

$\left\|\mathrm{x}_{2}-\mathrm{x}_{3}\right\|^{2}=\mathrm{P}_{\alpha}^{2}\left(\mathrm{x}_{2}, \mathrm{~T}_{2}\right) \leq \mathrm{D}^{2}\left(\mathrm{~T}_{1}, \mathrm{~T} \mathrm{x}_{2}\right)$

For $\mathrm{x}_{0} \in \mathrm{H}$ and $\mathrm{x}_{1} \in\left[\mathrm{T} \mathrm{x}_{0}\right]_{\alpha}$ with $\left(\mathrm{x}_{0}, \mathrm{x}_{1}\right) \geq 1$, by definition of fuzzy $\beta-$ admissible, we get $\beta\left(x_{1}, x_{2}\right) \geq 1$, then

$$
\left\|\mathrm{x}_{2}-\mathrm{x}_{3}\right\|^{2} \leq \mathrm{D}^{2}\left(\mathrm{~T} \mathrm{x}_{1}, \mathrm{Tx_{2 }}\right) \leq \beta\left(\mathrm{x}_{1}, \mathrm{x}_{2}\right) \mathrm{D}^{2}\left(\mathrm{~T} \mathrm{x}_{1}, \mathrm{~T} \mathrm{x}_{2}\right)
$$$$
\leq \Psi\left(\mathrm{M}\left(\mathrm{x}_{1}, \mathrm{x}_{2}\right)\right)
$$

$+\operatorname{Lmin}\left\{\mathrm{P}_{\alpha}^{2}\left(\mathrm{x}_{1}, \mathrm{~T}_{1}\right), \mathrm{P}_{\alpha}^{2}\left(\mathrm{x}_{2}, \mathrm{~T}_{2}\right), \mathrm{P}_{\alpha}^{2}\left(\mathrm{x}_{1}, \mathrm{~T}_{2}\right), \mathrm{P}_{\alpha}^{2}\left(\mathrm{x}_{2}, \mathrm{~T}_{1}\right)\right\}$ $\leq \Psi\left(\mathrm{M}\left(\mathrm{x}_{1}, \mathrm{x}_{2}\right)\right)$

$+\operatorname{Lmin}\left\{\mathrm{P}_{\alpha}^{2}\left(\mathrm{x}_{1}, \mathrm{x}_{2}\right), \mathrm{P}_{\alpha}^{2}\left(\mathrm{x}_{2}, \mathrm{x}_{3}\right), \mathrm{P}_{\alpha}^{2}\left(\mathrm{x}_{1}, \mathrm{x}_{3}\right), \mathrm{P}_{\alpha}^{2}\left(\mathrm{x}_{2}, \mathrm{x}_{2}\right)\right\}$

$\left\|\mathrm{x}_{2}-\mathrm{x}_{3}\right\|^{2} \leq \Psi\left(\mathrm{M}\left(\mathrm{x}_{1}, \mathrm{x}_{2}\right)\right)$.

By induction, we can construct a sequence $\left\{x_{n}\right\}$ in $H$ such that, for each $n \in N, x_{n} \in\left[T x_{n}\right]_{\alpha}$ with $\beta\left(x_{n-1}, x_{n}\right) \geq 1$ and

$$
\begin{aligned}
& \left\|x_{n}-x_{n+1}\right\|^{2} \leq \Psi\left(M\left(x_{n-1}, x_{n}\right)\right) . \\
& M\left(x_{n-1}, x_{n}\right)=\quad=\quad \frac{\alpha P_{\alpha}^{2}\left(x_{n-1}, T x_{n-1}\right) \cdot P_{\alpha}^{2}\left(x_{n}, T x_{n}\right)}{\left\|x_{n-1}-x_{n}\right\|^{2}}+ \\
& \beta\left\|x_{n-1}-x_{n}\right\|^{2}-\alpha P_{\alpha}^{2}\left(x_{n-1}, T x_{n-1}\right) \leq \\
& \frac{\alpha\left\|x_{n-1}-x_{n}\right\|^{2} \cdot\left\|x_{n+1}-x_{n}\right\|^{2}}{\left\|x_{n-1}-x_{n}\right\|^{2}}+\beta\left\|x_{n-1}-x_{n}\right\|^{2} \\
& \leq \alpha\left\|x_{n+1}-x_{n}\right\|^{2}+\beta\left\|x_{n-1}-x_{n}\right\|^{2}-\alpha\left\|x_{n+1}-x_{n}\right\|^{2} \\
& M\left(x_{n-1}, x_{n}\right) \leq \beta\left\|x_{n-1}-x_{n}\right\|^{2}
\end{aligned}
$$

We have $\left\|x_{n}-x_{n+1}\right\|^{2} \leq \Psi\left(\beta\left\|x_{n-1}-x_{n}\right\|^{2}\right)$ $\leq \Psi\left(\Psi\left(\beta\left\|x_{n-2}-x_{n-1}\right\|^{2}\right)\right)$ $\leq \Psi^{\mathrm{n}}\left(\beta \| x_{0}\right.$ $\mathrm{x} 12$.

Next, we will show that $\left\{x_{n}\right\}$ is a Cauchy sequence in $H$. Since continuous function $\Psi$, there exists $\epsilon>0$ and positive integer $\mathrm{h}=\mathrm{h}(\epsilon)$ such that $\sum_{\mathrm{n} \geq \mathrm{h}} \Psi^{\mathrm{n}}\left(\beta\left\|\mathrm{x}_{0}-\mathrm{x}_{1}\right\|^{2}\right)<\epsilon$.

Let $\mathrm{m}>n>h$.Using the triangular inequality, previous relation, we have $\left\|\mathrm{x}_{\mathrm{n}}-\mathrm{x}_{\mathrm{m}}\right\|^{2} \leq \sum_{\mathrm{k}=\mathrm{n}}^{\mathrm{m}-1} \beta\left\|\mathrm{x}_{\mathrm{k}}-\mathrm{x}_{\mathrm{k}+1}\right\|^{2} \leq$ $\mathrm{n} \geq \mathrm{h} \Psi \mathrm{n} \beta \mathrm{x} 0-\mathrm{x} 12<\epsilon$.

This implies that $\left\{x_{n}\right\}$ is a Cauchy sequence in $H$. By completeness of $\mathrm{H}$, there exists $\mathrm{x} \in \mathrm{H}$ such that $\mathrm{x}_{\mathrm{n}} \rightarrow$ $\mathrm{x}$ as $\mathrm{n} \rightarrow \infty$.

Finally, we show that $P_{\alpha}^{2}(x, T x)=0$. By condition (3), we have $\beta\left(x_{n}, x\right) \geq 1$, for all $n \in N$

Now we have $\mathrm{P}_{\alpha}^{2}(\mathrm{x}, \mathrm{Tx}) \leq\left\|\mathrm{x}_{\mathrm{n}+1}-\mathrm{x}_{\mathrm{n}}\right\|^{2}+\mathrm{P}_{\alpha}^{2}\left(\mathrm{x}_{\mathrm{n}+1}, \mathrm{Tx}\right)$

$$
\leq\left\|x_{n+1}-x_{n}\right\|^{2}+D_{\alpha}^{2}\left(T x_{n}, T x\right)
$$

$$
\begin{gathered}
\leq\left\|x_{n+1}-x_{n}\right\|^{2} \\
+\beta\left(x_{n}, x\right) D_{\alpha}^{2}\left(T x_{n}, T x\right) \\
\leq\left\|x_{n+1}-x_{n}\right\|^{2}+\Psi\left(\frac{\alpha P_{\alpha}^{2}\left(x_{n}, T x_{n}\right) \cdot P_{\alpha}^{2}(x, T x)}{\left\|x_{n}-x\right\|^{2}}\right. \\
\left.+\beta\left\|x_{n}-x\right\|^{2}-\alpha P_{\alpha}^{2}\left(x_{n}, T x_{n}\right)\right)
\end{gathered}
$$

$+\operatorname{Lmin}\left\{\mathrm{P}_{\alpha}^{2}\left(\mathrm{x}_{\mathrm{n}}, \mathrm{T} \mathrm{x}_{\mathrm{n}}\right), \mathrm{P}_{\alpha}^{2}(\mathrm{x}, \mathrm{Tx}), \mathrm{P}_{\alpha}^{2}\left(\mathrm{x}_{\mathrm{n}}, \mathrm{Tx}\right), \mathrm{P}_{\alpha}^{2}\left(\mathrm{x}, \mathrm{T} \mathrm{x}_{\mathrm{n}}\right)\right\}$

$$
\begin{gathered}
\leq\left\|x_{n+1}-x_{n}\right\|^{2} \\
+\Psi\left(\frac{\alpha\left\|x_{n+1}-x_{n}\right\|^{2} \cdot P_{\alpha}^{2}(x, T x)}{\left\|x_{n}-x\right\|^{2}}+\beta\left\|x_{n}-x\right\|^{2}\right. \\
\left.-\alpha\left\|x_{n+1}-x_{n}\right\|^{2}\right)
\end{gathered}
$$

$+\operatorname{Lmin}\left\{\| x_{\mathrm{n}+1}\right.$

$\left.-\mathrm{x}_{\mathrm{n}} \|^{2}, \mathrm{P}_{\alpha}^{2}(\mathrm{x}, \mathrm{Tx}), \mathrm{P}_{\alpha}^{2}\left(\mathrm{x}_{\mathrm{n}}, \mathrm{Tx}\right), \mathrm{P}_{\alpha}^{2}\left(\mathrm{x}, \mathrm{T} \mathrm{x}_{\mathrm{n}}\right)\right\}$

Letting $\mathrm{n} \rightarrow \infty$, it folloes that

$\mathrm{P}_{\alpha}^{2}(\mathrm{x}, \mathrm{Tx}) \leq \Psi(0)=0 \rightarrow \mathrm{P}_{\alpha}^{2}(\mathrm{x}, \mathrm{Tx})=0$.

Hence by lemma $2.8 \mathrm{x}_{\alpha} \subset \mathrm{Tx}$. This complete the proof.

Theorem 3.5: Let $\mathrm{H}$ be a Hilbert space and $\mathrm{T}$ be a fuzzy $\beta$ Jaggi contractive mapping satisfies the following conditions:

1) $T$ is fuzzy $\beta^{*}-$ admissible

2) There exists $\mathrm{x}_{0} \in \mathrm{H}$ and $\mathrm{x}_{1} \in\left[\mathrm{T} \mathrm{x}_{0}\right]_{\alpha}$ such that $\beta\left(x_{0}, x_{1}\right) \geq 1$

3) If $\left\{x_{n}\right\}$ is a sequence in $H$ such that $\beta\left(x_{n}, x_{n+1}\right) \geq 1$ and $\mathrm{x}_{\mathrm{n}} \rightarrow \mathrm{u}$ as $\mathrm{n} \rightarrow \infty$, then $\beta\left(\mathrm{x}_{\mathrm{n}}, \mathrm{u}\right) \geq 1$

4) $\Psi$ is continuous

Then, there exists $\mathrm{x} \in \mathrm{H}$ such that $\mathrm{x}_{\alpha}$ is a fuzzy fixed point of $\mathrm{T}$.

Proof: Trivial

In Theorem 3.4 and 3.5, we take $\Psi(\mathrm{t})=\theta \mathrm{t}$, where $\theta \in$ $(0,1)$, then we have the following corollary which is a fuzzy extension of fixed point theorem .

Corollary 3.6:Let $\mathrm{H}$ be a Hilbert space and $\mathrm{T}$ be a fuzzy mapping. Suppose that there exists $\beta: H \times H \rightarrow[0, \infty)$ such that $\beta(\mathrm{x}, \mathrm{y}) \mathrm{D}^{2}(\mathrm{Tx}, \mathrm{Ty}) \leq \theta(\mathrm{M}(\mathrm{x}, \mathrm{y}))+\mathrm{LN}(\mathrm{x}, \mathrm{y}), \forall \mathrm{x}, \mathrm{y} \in \mathrm{H}$ , where $\mathrm{L} \geq 0, \alpha \in[0,1], \beta>0$,

$$
\mathrm{M}(\mathrm{x}, \mathrm{y})=\frac{\alpha \mathrm{P}_{\alpha}^{2}(\mathrm{x}, \mathrm{Tx}) \cdot \mathrm{P}_{\alpha}^{2}(\mathrm{y}, \mathrm{Ty})}{\|\mathrm{x}-\mathrm{y}\|^{2}}+\beta\|\mathrm{x}-\mathrm{y}\|^{2}-\alpha \mathrm{P}_{\alpha}^{2}(\mathrm{x}, \mathrm{Tx})
$$




\section{International Journal of Science and Research (IJSR) \\ ISSN (Online): 2319-7064}

Index Copernicus Value (2015): 78.96 | Impact Factor (2015): 6.391

\section{And $\mathrm{N}(\mathrm{x}, \mathrm{y})$}

$\min \left\{\mathrm{P}_{\alpha}^{2}(\mathrm{x}, \mathrm{Tx}), \mathrm{P}_{\alpha}^{2}(\mathrm{y}, \mathrm{Ty}), \mathrm{P}_{\alpha}^{2}(\mathrm{x}, \mathrm{Ty}), \mathrm{P}_{\alpha}^{2}(\mathrm{y}, \mathrm{Tx})\right\}$.

Satisfies the following conditions:

1) $\mathrm{T}$ is fuzzy $\beta$ - admissible(or fuzzy $\beta^{*}-$ admissible)

2) There exists $\mathrm{x}_{0} \in \mathrm{H}$ and $\mathrm{x}_{1} \in\left[\mathrm{T} \mathrm{x}_{0}\right]_{\alpha}$ such that $\beta\left(x_{0}, x_{1}\right) \geq 1$

3) If $\left\{x_{n}\right\}$ is a sequence in $H$ such that $\beta\left(x_{n}, x_{n+1}\right) \geq 1$ and $x_{n} \rightarrow u$ as $n \rightarrow \infty$, then $\beta\left(x_{n}, u\right) \geq 1$

Then, there exists $x \in H$ such that $x_{\alpha}$ is a fuzzy fixed point of $\mathrm{T}$.

Proof: Trivial

\section{References}

[1] B.Mohammadi,S.Rezapour,N.Shahzad"Some results on fixed points of $\alpha-\Psi$ Ciric generalized multifunctions fixed point theory ,Appl.24(2013)

[2] Samet, B, Vetro, C, Vetro, P: Fixed point theorems for $\alpha$ $\psi$-contractive type mappings. Nonlinear Anal. 75, 21542165 (2012)

[3] L.A.zadeh , probability measures of fuzzy events, J.Math.Anal.Appl.23(1968),421-427

[4] S.Heilpern, Fuzzy mappings and fixed point theorem, J.Math. Anal.83(1981), 566-569.

[5] Estruch, VD, Vidal,A, "A note on fixed fuzzy points for fuzzy mappings",Rend. Ist. Mat. Univ. Trieste.32,3945(2001).

Volume 6 Issue 7, July 2017 www.ijsr.net 